Pesq. Vet. Bras. 36(3):204-208, março 2016

DOI:10.1590/S0100-736X2016000300009

\title{
Técnica de fatiamento do ovário para obtenção de oócitos em cutias (Dasyprocta prymnolopha) ${ }^{1}$
}

\author{
Maíra S. Ferraz ${ }^{2,5 *}$, Felipe de Jesus Moraes Júnior ${ }^{3}$, Matheus Levi T. Feitosa ${ }^{4}$, \\ Hatawa M. de Almeida ${ }^{5}$, Dayseanny de O. Bezerra ${ }^{5}$, Gerson T. Pessoa ${ }^{5}$, \\ Daniel M. de N. Albuquerque ${ }^{5,6}$ e Maria Acelina Martins de Carvalho ${ }^{7}$
}

\begin{abstract}
Ferraz M.S., Moraes Jr F.J., Feitosa M.L.T., Almeida H.M., Bezerra D.O., Pessoa G.T., Albuquerque D.M.N. \& Carvalho M.A.M. 2016. [Ovarian slicing technique to obtain oocytes from agoutis (Dasyprocta prymnolopha).] Técnica de fatiamento do ovário para obtenção de oócitos em cutias (Dasyprocta prymnolopha). Pesquisa Veterinária Brasileira 36(3):204-208. Departamento de Morfologia, Centro de Ciências da Saúde, Universidade Federal do Piauí, Teresina, PI 64049-550, Brazil. E-mail: mairasferraz@ufpi.edu.br

The reproductive biotechnologies are important tools for conservation of domestic and wild animal species, because they allow the recovery and future use of reproductive material. The aim of this study was to evaluate morphological parameters of COCs of agoutis obtained by slicing the ovary for using them in maturation and fertilization protocols in vitro production of embryos. Seventeen female agoutis NEPAS, CCA-UFPI, age and weight average of 3.9 years and $2.16 \mathrm{~kg}$, respectively, were underwent ovariossalpingohisterectomy. The ovaries after dissected and weighed on a precision balance were sliced individually. We proceeded the search and selection of COCs in stereomicroscope, which were identified and quantified by each ovary, and classified as to their morphology, by the quantity of layers of cumulus cells and the cytoplasm into four degrees. It has been found that the technique of slicing ovarian possible to obtain agouti COCs with recovery of loads and varying degrees of quality. There was no correlation between age and weight of animal ovaries, age and the number of COCs obtained; between the weight of agoutis and weight of ovaries and the number of COCs obtained.
\end{abstract}

INDEX TERMS: Ovarian slicing technique, oocytes, agoutis, Dasyprocta prymnolopha, rodent, cumulus-oocytes complex (COCs), IVM.

\footnotetext{
${ }^{1}$ Recebido em 10 de julho de 2015.

Aceito para publicação em 7 de novembro de 2015.

${ }^{2}$ Departamento de Morfologia, Centro de Ciências da Saúde, Universidade Federal do Piauí (UFPI), Campus Universitário Ministro Petrônio Portella, Bairro Ininga, Teresina, PI 64049-550, Brasil. *Autor para correspondência: mairasferraz@ufpi.edu.br

${ }^{3}$ Bolsista de Pós-Doutorado (PNPD, Programa de Mestrado em Ciência Animal, Universidade Estadual do Maranhão (UEMA), Cidade Universitária Paulo VI, São Luís, MA 65055-970, Brasil. E-mail: fmoraesjr@hotmail.com

${ }^{4}$ Bolsista de Pós-Doutorado (PNPD), Centro de Ciências Agrárias (CCA), UFPI, Campus Universitário Ministro Petrônio Portella, Bairro Ininga, Teresina, PI 64049-550, Brasil. E-mail: mtajra@gmail.com

${ }^{5}$ Pós-Graduando em Ciência Animal, CCA, UFPI, Campus Universitário Ministro Petrônio Portella, Bairro Ininga, Teresina, PI 64049-550, Brasil.

${ }^{6}$ Instituto Federal de Educação, Ciência e Tecnologia do Piauí (IFPI), Campus Uruçuí, Rodovia PI 247, Km 7, s/n, Portal dos Cerrados, Uruçuí, PI 64860-000, Brasil. E-mail: daniel.albuquerque@ifpi.edu.br

${ }^{7}$ Departamento de Morfofisiologia Veterinária, Centro de Ciências Agrárias, UFPI, Campus Universitário Ministro Petrônio Portella, Bairro Ininga, Teresina, PI 64049-550, Brasil. E-mail: mcelina@ufpi.edu.br
}

RESUMO.- As biotécnicas da reprodução são importantes ferramentas para conservação de espécies domésticas e silvestres, pois permitem a recuperação e uso futuro de material reprodutivo. 0 objetivo deste estudo foi avaliar parâmetros morfológicos de CCOs de cutias, obtidos pela técnica de fatiamento do ovário para utilização em protocolos de maturação e fecundação na produção in vitro de embriões. Foram utilizadas dezessete cutias fêmeas do NEPAS, CCA- UFPI, com idade e peso médios de 3,9 anos e $2,16 \mathrm{Kg}$, respectivamente, que foram submetidas à ovariossalpingohisterectomia. Os ovários após dissecados e pesados em balança de precisão, foram fatiados individualmente. Procedeu-se a busca e seleção dos CCOs em estereomicroscópio, os quais foram identificados e quantificados por cada ovário, além de classificados quanto a sua morfologia segundo a quantidade de camadas de células do cumulus e ao citoplasma em quatro graus. Verificou-se que a técnica de fatiamento do ovário possibilitou a obtenção 
de CCOs de cutias, com recuperação de grande quantidade e de variados graus de qualidade. Não houve correlações entre a idade dos animais e o peso dos ovários; a idade e o número de CCOs obtidos; entre o peso das cutias e o peso dos ovários e o número de CCOs obtidos.

TERMOS DE INDEXAÇÃO: Ovário, oócitos, cutias, Dasyprocta prymnolopha, roedor, complexos cumulus-oócito (CCOs), MIV.

\section{INTRODUÇÃO}

As biotécnicas da reprodução são importantes ferramentas para conservação de espécies silvestres visto que permitem a recuperação e o uso futuro de material reprodutivo, além de facilitar a troca de gametas entre populações, evitando os efeitos negativos da endogamia, bem como as incompatibilidades comportamentais entre indivíduos que inviabilizam a reprodução natural. Entretanto, no contexto da reprodução assistida, uma fonte de obtenção de oócitos é crucial para preservação de recursos genéticos, conservação de espécies ameaçadas, transgênese e clonagem, por transferência nuclear, para propósitos reprodutivos ou para geração de células-tronco embrionárias para serem utilizadas em medicina regenerativa. A obtenção destas células constitui-se em um requisito importante tanto para estudos básicos sobre reprodução quanto para pesquisas em técnicas de reprodução assistida, como a produção in vitro de embriões. 0 sucesso dessa biotecnologia depende, principalmente, da qualidade oocitária que está diretamente relacionada à presença das células do cumulus e às peculiaridades dos métodos de recuperação dos complexos cumulus-oócito (CCOs).

Várias técnicas para obtenção de CCOs podem ser utilizadas e possuem impacto considerável sobre a quantidade e a morfologia dos CCOs recuperados e, consequentemente, sobre a competência no desenvolvimento oócitário (Bols et al. 1997). Os CCOs podem ser obtidos da tuba uterina, logo após a ovulação, sendo que neste caso, o processo de maturação oocitária já se iniciou in vivo, ou de folículos pré-ovulatórios, imaturos e por vezes atrésicos, havendo necessidade de ser realizada a maturação oocitária in vitro (Wani 2002). Os métodos de coleta mais comumente utilizados para obtenção de CCOs ainda não maturados são: aspiração folicular, fatiamento do ovário (slicing) e curetagem da parede folicular (scraping). De acordo com Wani et al. (2000), a técnica de slicing ovariano possibilita o acesso a folículos localizados profundamente dentro do córtex do ovário, resultando na recuperação de maior quantidade de CCOs do que o método da aspiração folicular com agulha acoplada à seringa, no qual ainda há maior possibilidade de perda dos mesmos. Assim, esta técnica tem grande aplicabilidade às pesquisas por constituírem uma fonte abundante de CCOs (Gibbons et al. 2008).

Há procedimentos padronizados para coleta de CCOs em várias espécies domésticas e silvestres, em roedores de laboratório (Nagy et al. 2002) e alguns ratos silvestres (Martín-Coello et al. 2008), mas estes procedimentos nem sempre podem ser aplicados às demais espécies, e isto, por sua vez, gera a necessidade de desenvolvimento de técnicas de coletas adaptadas à espécie a ser trabalhada. Deste modo, diante da escassez de informações sobre técnicas para obtenção de CCOs de cutias, com vista à utilização na produção in vitro de embriões, o objetivo deste trabalho foi avaliar a técnica do fatiamento do ovário para a obtenção de CCOs de cutias, analisando parâmetros morfológicos, além da quantidade e qualidade dos CCOs obtidos, bem como a existência de correlações entre a idade e o peso das cutias e o número de oócitos obtidos.

\section{MATERIAL E MÉTODOS}

O estudo foi realizado no Centro de Ciências Agrárias (CCA) da Universidade Federal do Piauí (UFPI), em Teresina, PI. Foram utilizadas dezessete cutias fêmeas oriundas do Núcleo de Estudos e Preservação de Animais Silvestres (NEPAS) da UFPI (Registro de Criadouro IBAMA no 02/08 - 618), com idade e peso médios de 3,9 anos e $2,16 \mathrm{~kg}$, respectivamente.

Inicialmente, as cutias foram submetidas a exame ultrassonográfico para excluir os animais gestantes do experimento. As fêmeas não gestantes foram alojadas em baias onde ficaram isoladas dos machos durante todo o experimento. Os animais foram novamente submetidos ao exame ultrassonográfico 30 dias após o primeiro exame para confirmar o diagnóstico negativo de gestação.

Os animais receberam como alimentação ração comercial peletizada (proteína bruta mínima - 12\%, extrato etéreo - 1,5\%, matéria fibrosa - $12 \%$, matéria mineral - $13 \%$, cálcio - 1,3\% e fósforo - $0,4 \%$ ), além de milho, frutas e verduras regionais da época e água ad libitum.

As cutias foram contidas manualmente com auxílio de puçá e então levadas ao Laboratório de Pesquisas Morfológicas em Ciência Animal do CCA/UFPI, onde foram submetidas à aplicação da medicação pré-anestésica (meperidina $2 \mathrm{mg} / \mathrm{Kg}$, via intramuscular profunda). Após 15 minutos foram aplicados $50 \mathrm{mg} / \mathrm{kg}$ de cloridrato de quetamina associados a $1 \mathrm{mg} / \mathrm{kg}$ de cloridrato de xilazina por via intramuscular para indução anestésica. Posteriormente, foi realizada a tricotomia do abdômen da cutia seguida da assepsia local com álcool a 70\% e iodo povidona para que se procedesse a ovariossalpingohisterectomia (OSH). Após o procedimento cirúrgico as cutias retornaram ao NEPAS, onde permaneceram isoladas dos demais animais e receberam medicação anti-inflamatória (meloxicam 0,1mg/kg, via subcutânea) por 5 dias e antibioticoterapia (enrofloxacina $10 \mathrm{mg} / \mathrm{kg}$, via subcutânea) por 7 dias.

Os ovários foram cuidadosamente dissecados e pesados individualmente em balança de precisão. Após este procedimento os ovários foram transportados até o Laboratório de Biotecnologia da Reprodução Animal no CCA/UFPI, em tubo de centrifuga de $15 \mathrm{~mL}$ contendo $10 \mathrm{~mL}$ de solução salina tamponada com fosfato (PBS) à temperatura de $37^{\circ} \mathrm{C}$. Seguidamente, os ovários foram fatiados individualmente com lâmina de bisturi em placa de petri de 100x15mm (TPP). Após o fatiamento procedeu-se a procura e seleção dos CCOs em estereomicroscópio (Nikon SMZ 645). Os CCOs foram identificados, quantificados por cada ovário e colocados no meio de manutenção (TQC Holding Plus ${ }^{\circledR}$ ) e classificados quanto a sua morfologia segundo a quantidade de camadas de células do cumulus e ao citoplasma em quatro diferentes graus de acordo com Leibfried \& First, (1979).

Foi realizada a análise descritiva de todos os parâmetros avaliados (idade e peso do animal, peso dos ovários direito e esquerdo e número de CCOs obtidos nos ovários esquerdo e direito, bem como o número total de CCOs por animal). Realizamos o teste t de Student para as variáveis de peso dos ovários e número de CCOs dos ovários direito e esquerdo. Para a avaliação da qualidade dos CCOs, foi feita uma análise de proporção entre os diferentes tipos 
obtidos. A avaliação de correlações entre idade e peso do animal, idade do animal e peso dos ovários, idade do animal e número de CCOs obtidos, peso do animal e peso dos ovários, peso do animal e número de CCOs obtidos e peso dos ovários e número de CCOs foi feita pelo teste de correlação de Pearson e teste t de Student para testar a significância das correlações. A análise estatística foi realizada utilizando-se o programa "Statistical Analysis System" (SAS 2002) por meio dos procedimentos PROC MEANS, PROC TTEST, PROC CORR, com $\mathrm{P}<0,01$.

Todos os procedimentos realizados neste trabalho foram autorizados pelo Comitê de Ética em Experimentação Animal da Universidade Federal do Piauí (protocolo no 001/13 CEEA/PI) e pelo Instituto Chico Mendes de Conservação da Biodiversidade (ICMBio) por meio do Sistema de Autorização e Informação em Biodiversidade (SISBIO) de número de protocolo 36942-1.

\section{RESULTADOS}

As cutias apresentaram, em média, 3,9 $\pm 1,2$ anos e pesaram $2,1 \pm 0,3 \mathrm{~kg}$. Os ovários direito e esquerdo pesaram, em média, 74 e $75 \mathrm{mg}$, respectivamente.

Os resultados da coleta de CCOs por fatiamento dos ovários estão apresentados no Quadro 1. Foi obtida uma média de 8,5 CCOs por ovário, ou 17,0 CCOs por cutia. Não foram verificadas diferenças significativas entre os pesos dos ovários direito e esquerdo $(\mathrm{P}>0,05)$; e também entre o número de CCOs obtidos dos ovários direito e esquerdo $(\mathrm{P}>0,05)$ pelo teste t de Student a 5\% de probabilidade.

Os CCOs de cutia obtidos apresentaram diferentes graus de qualidade, sendo que aqueles que apresentaram cumulus compacto presente, contendo mais de três camadas de células; o ooplasma com granulações finas e homogêneas, preenchendo o interior da zona pelúcida e de coloração marrom foram classificados como grau I $(3,5 \%)$. Aqueles CCOs que apresentaram cumulus compacto parcialmente presente em volta do oócito ou rodeando completamente o oócito, com menos de três camadas celulares; o ooplasma apresentava granulações distribuídas heterogeneamente, estando mais concentradas no centro e mais claras na periferia ou condensadas em um só local aparentando uma mancha escura, foram classificados como grau II $(6,2 \%)$. Os CCOs com cumulus presente, mas expandido; o ooplasma contraído com espaço entre a membrana celular e a zona pelúcida, preenchendo irregularmente o espaço perivitelino foram classificados como grau III $(68,2 \%)$. E os CCOs sem cumulus foram classificados como grau IV $(13,5 \%)$. Foram também observados CCOs pequenos e de coloração escura com citoplasma irregular, que foram classificados como degenerados $(8,6 \%)$.

As correlações de Pearson entre as características avaliadas foram em grande parte não significativas $(\mathrm{P}>0,01)$, positivas e de alta à média magnitude (Quadro 2).

A idade das cutias apresentou correlação positiva significativa apenas com o peso do animal $(0,66)$. Não foram observadas correlações significativas entre a idade do animal e o peso dos ovários e nem entre a idade do animal e o número de CCOs obtidos.

Com relação aos pesos das cutias, não foram observadas correlações significativas entre o peso da cutia e o número CCOs obtidos. Entre o peso da cutia e o peso dos ovários foi possível observar, que apesar de não significativa, existe
Quadro 1. Peso e idade das cutias (Dasyprocta prymnolopha), peso dos ovários e número de CCOs obtidos

\begin{tabular}{cccccccc}
\hline Animal & $\begin{array}{c}\text { Idade } \\
\text { cutia } \\
\text { (anos) }\end{array}$ & $\begin{array}{c}\text { Peso } \\
\text { cutia } \\
\text { (Kg) }\end{array}$ & $\begin{array}{c}\text { Peso } \\
\text { OD } \\
\text { (mg) }\end{array}$ & $\begin{array}{c}\text { Peso } \\
\text { OE } \\
\text { (mg) }\end{array}$ & $\begin{array}{c}\text { N. CCOs } \\
\text { OD }\end{array}$ & N. CCOs & $\begin{array}{c}\text { Total de } \\
\text { CCOs/ ovário } \\
\text { de cutia }\end{array}$ \\
\hline 1 & 4,6 & 2,1 & 103 & 64,3 & 16 & 16 & 32 \\
2 & 6,0 & 2,3 & 82,2 & 93,8 & 17 & 10 & 27 \\
3 & 3,1 & 2,1 & 71,8 & 95,1 & 17 & 9 & 26 \\
4 & 5,1 & 2,4 & 94,0 & 52,6 & 6 & 5 & 11 \\
5 & 2,8 & 1,7 & 55,1 & 85,2 & 22 & 6 & 28 \\
6 & 3,1 & 2,3 & 44,9 & 40,7 & 5 & 12 & 17 \\
7 & 4,5 & 2,3 & 40,4 & 35,6 & 3 & 6 & 9 \\
8 & 5,0 & 2,2 & 108,5 & 98,8 & 12 & 4 & 16 \\
9 & 2,8 & 2,3 & 13,4 & 18,5 & 2 & 1 & 3 \\
10 & 3,2 & 2,1 & 56,6 & 48 & 5 & 8 & 13 \\
11 & 4,7 & 2,2 & 68,9 & 65 & 10 & 9 & 19 \\
12 & 4,5 & 2,2 & 65,9 & 82,5 & 6 & 10 & 16 \\
13 & 1,2 & 1,1 & 99,6 & 85,3 & 1 & 1 & 2 \\
14 & 3,0 & 2,1 & 95,3 & 81,9 & 6 & 11 & 17 \\
15 & 3,6 & 2,3 & 78,0 & 83,6 & 7 & 11 & 18 \\
16 & 4,4 & 2,1 & 92,2 & 121,4 & 6 & 9 & 15 \\
17 & 5,2 & 2,2 & 94,8 & 122,3 & 11 & 9 & 20 \\
Totais & - & - & - & - & 152 & 137 & 289 \\
Médias & 3,9 & 2,1 & 74 & 75 & 8,9 & 8,0 & 17 \\
Desvios- & 1,2 & 0,3 & 26 & 29 & 6,0 & 3,8 & 8,2 \\
padrão & & & & & & & \\
CV (\%) & 30,7 & 14,2 & & 38,7 & 67,3 & 48,1 & 48,42 \\
Erro-padrão & 0,2 & 0,1 & 6,3 & 7,0 & 1,4 & 0,9 & 1,9 \\
& & & & & & &
\end{tabular}

Quadro 2. Estimativas dos coeficientes de correlação de

Pearson entre a idade e o peso das cutias (Dasyprocta prymnolopha), idade e peso dos ovários, idade e número de CCOs obtidos, peso dos animais e peso dos ovários, peso dos animais e número de CCOs obtidos e pesos dos ovários direito e esquerdo e número de CCOs obtidos por fatiamento dos ovários direito e esquerdo de cutias

\begin{tabular}{lccccc}
\hline & Peso corporal & PT & NOD & NOE & TO \\
\hline Idade & $0.6642^{* *}$ & $0.2843^{\text {ns }}$ & $0.3159^{\text {ns }}$ & $0.3455^{\text {ns }}$ & $0.3942^{\text {ns }}$ \\
PESO & 1 & $-0.2449^{\text {ns }}$ & $0.0046^{\text {ns }}$ & $0.3743^{\text {ns }}$ & $0.1798^{\text {ns }}$ \\
POD & & $0.9132^{* * *}$ & $0.2359^{\text {ns }}$ & $0.2326^{\text {ns }}$ & $0.2823^{\text {ns }}$ \\
POE & & $0.9311^{* * *}$ & $0.4244^{\text {ns }}$ & $0.1854^{\text {ns }}$ & $0.3981^{\text {ns }}$ \\
PT & & 1 & $0.3635^{\text {ns }}$ & $0.2251^{\text {ns }}$ & $0.3722^{\text {ns }}$ \\
NOD & & & 1 & $0.3503^{\text {ns }}$ & $0.8972^{* * *}$ \\
NOE & & & & 1 & $0.7279^{* * *}$ \\
TO & & & & & 1
\end{tabular}

$\overline{\mathrm{POD}}$ = Peso ovário direito; $\mathrm{POE}$ = Peso ovário esquerdo; PT = Peso Total; NOD = Número de CCOs OD; NOE = Número de CCOs OE; TO = total de CCOs/cutia; Mediaoo = Média de CCOs/ovário. ${ }^{* *} \mathrm{P}<0,01 ;{ }^{* * *} \mathrm{P}<0,001 ;{ }^{\text {ns }}$ = não-significativo. Teste t de Student.

uma relação negativa entre eles. Os pesos ovarianos direito e esquerdo não apresentaram correlação significativa com o número de CCOs obtidos dos ovários direito e esquerdo, respectivamente.

\section{DISCUSSÃO}

A média de CCOs obtida neste trabalho mostrou-se superior ao número de CCOs obtidos, também por fatiamento do ovário, em chinchilas (Aiudi et al. 2007).

De acordo com Wani et al. (2000), a técnica de fatiamento do ovário possibilita o acesso a folículos localizados profundamente dentro do córtex do ovário, resultando na recuperação de maior quantidade de CCOs do que o método da aspiração folicular com agulha acoplada à seringa, no 
qual ainda há maior possibilidade de perda dos mesmos. Entretanto, neste estudo em cutias observou-se que a média obtida para recuperação de CCOs por fatiamento do ovário foi semelhante à mostrada por Martín-Coello et al. (2008) em M. spretus (19,3), superior à obtida também por estes autores em M. spicilegus $(11,2)$ e inferiores à média obtida em M. musculus $(25,7)$. Entretanto, apesar de obter médias superiores naquele trabalho, as três espécies do estudo foram submetidas a tratamento hormonal anterior, o que provavelmente estimulou o desenvolvimento folicular e gerou uma maior média de CCOs obtidos. Neste experimento os autores verificaram ainda que a média de CCOs obtida por aspiração folicular foi maior que a média de ooócitos obtidos da tuba uterina após a superovulação nas três espécies trabalhadas.

No que diz respeito às características dos ovários das cutias, foi possível verificar que os resultados obtidos se aproximam aos encontrados por Guimarães, Matos \& Vale (1994) e por Almeida et al. (2003) para a cutia, e aos de Matamoros (1981) para a paca, mas são inferiores aos expostos por Weir (1971), que encontrou um peso médio de $0,207 \mathrm{~g}$, para a cutia.

Embora a aparência não assegure a capacidade dos CCOs de se desenvolverem in vitro, este parâmetro morfológico serve como indicador de sua viabilidade. Isso porque os CCOs dependem do suporte adequado das células do cumulus, que têm ligação direta com o ooplasma, permitindo o transporte de nutrientes e sinais que controlam o metabolismo e a maturação nuclear e citoplasmática (Hirshfield 1991). Desta forma, a avaliação visual quanto à compactação, quantidade e aparência das células do cumulus e uniformidade do citoplasma tem sido utilizada como meio de selecionar CCOs e classificá-los qualitativamente (Pavlok et al. 1992, Moreno et al. 1993, Costa et al. 1997).

Em estudos com bovinos, Costa et al. (1997) observaram que a maior porcentagem de CCOs obtidos após aspiração folicular foram aqueles que apresentavam corona radiata intacta e mais de três camadas compactas de células do cumulus-oócito, classificado no nosso estudo como grau I. A diferença observada entre os dois estudos deve-se às diferentes fases do ciclo estral em que os animais encontravam-se no momento dos experimentos, visto que alguns estudos indicam que a progesterona plasmática afeta a qualidade oocitária (Hendriksen et al. 2004, Pfeifer et al. 2009, Pfeifer et al. 2011) pois oócitos coletados na fase tardia de diestro são mais competentes que aqueles coletados no início do diestro ou na fase folicular do ciclo estral, onde a concentração de progesterona é mais baixa (Machatkova et al. 1996, 2004).

Os resultados obtidos neste trabalho diferem dos obtidos por Reis et al. (2006), que observaram que o número de CCOs produzidos foi diretamente proporcional à idade das vacas. Estes autores estudaram também os efeitos da idade das vacas na qualidade dos CCOs e relataram que há uma correlação positiva entre estes parâmetros até os 7-8 anos, começando a baixar a partir desta idade. Assim, tal diferença verificada entre os estudos pode dever-se, em parte, pela diferença dos intervalos de idade das espécies estudadas, bem como pelas diferenças fisiológicas entre as mesmas.
A ausência de correlação entre os pesos ovarianos direito e esquerdo e o número de CCOs obtidos dos ovários direito e esquerdo, observada neste estudo, difere do observado para outras espécies, como demonstrado em bovinos havendo maior correlação do peso ovariano com o total de CCOs (Fernandes et al. 2001).

\section{CONCLUSÕES}

Assim, é possível concluir que, a técnica de fatiamento do ovário é viável para obtenção de CCOs de cutias, permitindo a recuperação de grande quantidade de CCOs, de variados graus de qualidade.

Analisando as correlações entre a idade dos animais e o peso dos ovários, a idade e o número de CCOs obtidos, bem como entre o peso das cutias e o peso dos ovários e o número de CCOs obtidos, não houve diferenças significativas.

\section{REFERÊNCIAS}

Aiudi G., Cinone M., Maritato F., Sandro Salvati A. \& Dell’Aquila M.E. 2007. Rescueand in vitro maturation of follicular oocytes in Chinchilla laniger. Reprod. Fertil. Dev. 19:119-324.

Almeida M.M., Carvalho M.A.M., Cavalcante Filho M.F., Miglino M.A. \& Menezes D.J.A. 2003. Estudo morfológico e morfométrico do ovário de cutias (Dasyprocta aguti, Linnaeus, 1766). Braz. J. Vet. Res. Anim. Sci. 40:55-62.

Bols P.E.J., Ysebaert M.T., Van Soom A. \& De Kruif A. 1997. Effects of needle tip bevel and aspiration procedure on the morphology and developmental capacity bovine compact cumulus oocyte complexes. Theriogenology 47:1221- 1236 .

Costa E.P., Vale Filho V.R., Nogueira J.C., Sá W.F. \& Costa A.H.A. 1997. Tipos morfológicos de oócitos bovinos. Arq. Bras. Med. Vet Zootec. 49(4):417-424.

Fernandes C.E., Dode M.A.N., Godoy K. \& Rodovalho N. 2001. Efeito estacional sobre características ovarianas e produção de oócitos em vacas Bos indicus no Mato Grosso do Sul. Braz. J. Vet. Res. Anim. Sci. 38(3):131-135.

Gibbons A., Bonnet F.P., Cueto M.I., Salamone D. \& Catala M. 2008. Colheita de oócitos guiada por laparoscopia em caprinos e ovinos. Acta Scient. Vet. 36(Supl.2):223-230.

Guimarães D.A., Matos E. \& Vale W.G. 1994. Estudo morfológico do sistema genital feminino de cutia (Dasyprocta prymnolopha, Rodentia: Cavidae). Revta Bras. Ciênc. Morfol. 11(2):167-171.

Hendriksen P.J., Steenweg W.N., Harkema J.C., Merton J.S., Bevers M.M., Vos P.L. \& Dieleman S.J. 2004. Effect of different stages of the follicular wave on in vitro development competence of bovine oocytes. Theriogenology 61:909-920.

Hirshfield A.N. 1991. Development of follicles in the mammalian ovary. Int. Rev. Cytol. 124:43-101.

Leibfried-Rutledge M.L. \& First N.L. 1979. Characterization of bovine follicular oocytes and their ability to mature in vitro. J. Anim. Sci. 48:76-86.

Machatkova M., Jokesova E., Petelikova J \& Dvoracek V. 1996. Developmental competence of bovine embryos derived from oocytes collected at various stages of the estrous cycle. Theriogenology. 45: 801-810.

Machatkova M., Krausova K., Jokesova E \& Tomanek M. 2004. Developmental competence of bovine oocytes: effects of follicle size and the phase of follicular wave on in vitro embryo production. Theriogenology. 61:329-335.

Matamoros Y. 1981. Anatomía e histología del sistema reproductor del tepezcuinte (Cuniculus paca). Revta Biol. Trop. 29(1):155-164.

Martín-Coello J., Gonzalez R., Crespo C., Gomendio M. \& Roldan E.R. 2008. Superovulation and in vitro oocyte maturation in three species of mice (Mus musculus, Mus spretus and Mus spicilegus). Theriogenology 70:1004-1013.

Moreno J.F., Flores-Foxworth G., Westhusin M. \& Kraemer D.C. 1993. Influence of pregnancy and presence of a CL on quantity and quality of bovine oocytes obtained from ovarian follicles aspirated post-mortem. Theriogenology 39(1):271. 
Nagy A., Gertsenstein M., Vintersten K. \& Behringer T. 2002. Manipulating the Mouse Embryo: a laboratory manual. 3rd ed. Cold Spring Harbor Laboratory Press, Cold Spring Harbor.

Pavlok A., Lucas-Hahn A. \& Nemann H. 1992. Fertilization and developmental competence of bovine oocytes derived from different categories of antral follicles. Mol. Reprod. Devel. 31(1):63-67.

Pfeifer L.F.M., Sartori R., Pivato I., Rumpf R., Nogueira G.P., Xavier E., Dionello N.J. \& Correa M.N. 2009. Effect of circulating progesterone on in vitro developmental competence of bovine oocytes. Anim. Reprod. 6:473-478.

Pfeifer L.F.M., Campos H., Miguel Jr J.C., Silveira L.L., Schneider A., Correa M.N. \& Rumpf R. 2011. Aumento da qualidade de ovócitos recuperados por punção folicular de vacas submetidas previamente à superovulação. Revta Bras. Reprod. Anim. 35:363-367.
Reis A., Metelo R., Santos P. \& Silva F.M. 2006. Efeito da estrutura ovárica e da idade de bovinos da raça Holstein Friesian na quantidade e qualidade de ovócitos e de embriões produzidos in vitro. Braz. J. Vet. Res. Anim. Sci. 43(5):629-636.

Statistical Analysis System - SAS. 2002. User's guide. Cary, SAS Institute. $525 p$.

Wani N.A. 2002. In vitro maturation and in vitro fertilization of sheep oocytes. Small Rumin. Res. 44:89-95.

Wani N.A., Wani G.M., Khan M.Z. \& Salahudin S. 2000. Effect of oocyte harvesting techniques on in vitro maturation and in vitro fertilization in sheep. Small Rumin. Res. 36:63-67.

Weir B. 1971. Some observations on reproduction in the female agouti, Dasyprocta aguti. J. Reprod. Fert. 24(2):203-211. 